TRANSACTIONS OF THE

AMERICAN MATHEMATICAL SOCIETY

Volume 362, Number 5, May 2010, Pages 2525-2540

S 0002-9947(09)04876-4

Article electronically published on December 11, 2009

\title{
PFAFFIAN PRESENTATIONS OF ELLIPTIC NORMAL CURVES
}

\author{
TOM FISHER
}

\begin{abstract}
We investigate certain alternating matrices of linear forms whose Pfaffians generate the homogeneous ideal of an elliptic normal curve, or one of its higher secant varieties.
\end{abstract}

\section{INTRODUCTION}

We work over an algebraically closed field $k$ of arbitrary characteristic. An elliptic normal curve $C \subset \mathbb{P}^{n-1}$ is a smooth curve of genus one and degree $n$ that is contained in no hyperplane. The $r$ th higher secant variety $\operatorname{Sec}^{r} C$ is the Zariski closure of the locus of all $(r-1)$-planes spanned by $r$ points on $C$. As special cases we have $\operatorname{Sec}^{0} C=\emptyset, \operatorname{Sec}^{1} C=C$ and $\operatorname{Sec}^{2} C=\operatorname{Sec} C$. It is shown in $\mathrm{L}$ that $\operatorname{Sec}^{r} C$ is an irreducible variety of codimension $\max (n-2 r, 0)$. Moreover it is shown in vBH, Proposition 8.15] that if $n \geq 2 r+1$, then $\operatorname{Sec}^{r} C$ has singular locus $\operatorname{Sec}^{r-1} C$. Thus an elliptic normal curve is uniquely determined by any one of its higher secant varieties that is not the whole of projective space.

We write $I(X)$ for the homogeneous ideal of a projective variety $X$. By convention $I(\emptyset)$ is the irrelevant ideal. It is well known that if $n \geq 4$, then $I(C)$ is generated by a vector space of quadrics of dimension $n(n-3) / 2$. We recall the generalisation of this result to higher secant varieties.

Proposition 1.1. If $n \geq 2 r+2$, then $I\left(\operatorname{Sec}^{r} C\right)$ is generated by a vector space of $(r+1)$-ics of dimension $\beta(r+1, n)$ where

$$
\beta(r, n)=\left(\begin{array}{c}
n-r \\
r
\end{array}\right)+\left(\begin{array}{c}
n-r-1 \\
r-1
\end{array}\right)
$$

is the number of ways of choosing $r$ elements from $\mathbb{Z} / n \mathbb{Z}$ such that no two elements are adjacent.

Proof. This is a special case of Theorem 4.1

Let $\mathcal{L}(D)=H^{0}(C, \mathcal{O}(D))$ be the Riemann-Roch space determined by a divisor $D$ on $C$. Let $H$ be the divisor of a hyperplane section, and let $D_{1}, D_{2}$ be divisors on $C$ with $D_{1}+D_{2}=H$. We write $\Phi\left(D_{1}, D_{2}\right)$ for the matrix of linear forms representing the multiplication map

$$
\mathcal{L}\left(D_{1}\right) \times \mathcal{L}\left(D_{2}\right) \rightarrow \mathcal{L}(H) .
$$

It is clear that $\Phi\left(D_{1}, D_{2}\right)$ has rank at most 1 on $C$, and so has rank at most $r$ on $\operatorname{Sec}^{r} C$.

Received by the editors June 1, 2006, and in revised form, March 17, 2008.

2010 Mathematics Subject Classification. Primary 14H52; Secondary 14M12.

(C)2009 American Mathematical Society 
Definition 1.2. A matrix of linear forms is a determinantal presentation of $\operatorname{Sec}^{r} C$ if its $(r+1) \times(r+1)$ minors generate $I\left(\operatorname{Sec}^{r} C\right)$.

Determinantal presentations for curves of arbitrary genus have been studied in EKS. A set-theoretic generalisation to higher secant varieties is given in Ra. In \$2 we establish the following analogue of the main result of [EKS for higher secant varieties of elliptic normal curves.

Theorem 1.3. Let $n \geq 2 r+1$. Then $\Phi\left(D_{1}, D_{2}\right)$ is a determinantal presentation of $\operatorname{Sec}^{r} C$ if and only if

(i) $\operatorname{deg} D_{1}, \operatorname{deg} D_{2} \geq r+2$, and

(ii) if $\operatorname{deg} D_{1}=\operatorname{deg} D_{2}=r+2$, then $D_{1} \nsim D_{2}$.

We fit Theorem 1.3 into a bigger picture involving rank 2 vector bundles $\mathcal{E}$ on $C$ with $\operatorname{det} \mathcal{E} \simeq \mathcal{O}(1)$. Here $\mathcal{O}(1)$ is the line bundle on $C$ associated to the hyperplane section. We write $\Phi(\mathcal{E})$ for the alternating matrix of linear forms representing the determinant map

$$
\bigwedge^{2} H^{0}(C, \mathcal{E}) \rightarrow H^{0}(C, \mathcal{O}(1))=\mathcal{L}(H) .
$$

It is clear that $\Phi(\mathcal{E})$ has rank at most 2 on $C$, and so has rank at most $2 r$ on $\operatorname{Sec}^{r} C$. The analogue of Definition 1.2 is

Definition 1.4. An alternating matrix of linear forms is a Pfaffian presentation of $\operatorname{Sec}^{r} C$ if its $(2 r+2) \times(2 r+2)$ Pfaffians generate $I\left(\operatorname{Sec}^{r} C\right)$.

For $\mathcal{E}$ decomposable the situation is not new. Indeed we can write $\mathcal{E} \simeq \mathcal{O}\left(D_{1}\right) \oplus$ $\mathcal{O}\left(D_{2}\right)$, where $D_{1}, D_{2}$ are divisors on $C$ with $D_{1}+D_{2}=H$. Then with respect to suitable bases we have

$$
\Phi(\mathcal{E})=\left(\begin{array}{cc}
0 & \Phi\left(D_{1}, D_{2}\right) \\
-\Phi\left(D_{1}, D_{2}\right)^{T} & 0
\end{array}\right) .
$$

So $\Phi(\mathcal{E})$ is a Pfaffian presentation of $\operatorname{Sec}^{r} C$ if and only if $\Phi\left(D_{1}, D_{2}\right)$ is a determinantal presentation of $\operatorname{Sec}^{r} C$.

A theorem of Atiyah (Proposition 6.1) says that for $n$ odd there is a unique indecomposable rank 2 vector bundle $\mathcal{E}$ on $C$ with $\operatorname{det} \mathcal{E} \simeq \mathcal{O}(1)$. By the vector bundle form of Riemann-Roch (recalled in $₫$ ) we have $\operatorname{dim} H^{0}(C, \mathcal{E})=n$. Our main result is the following analogue of Theorem 1.3 .

Theorem 1.5. Let $n \geq 3$ be an odd integer. Let $C$ be an elliptic normal curve of degree $n$, and let $\mathcal{E}$ be the unique indecomposable rank 2 vector bundle on $C$ with $\operatorname{det} \mathcal{E} \simeq \mathcal{O}(1)$. Then

(i) The $n$ submaximal Pfaffians of $\Phi(\mathcal{E})$ are linearly independent.

(ii) If $n \geq 2 r+3$, then $\Phi(\mathcal{E})$ is a Pfaffian presentation of $\operatorname{Sec}^{r} C$.

The proof of Theorem 1.5(ii) from Theorem 1.5)(i) is an induction similar to the proof of Theorem 1.3. To prove Theorem 1.5(i) it is helpful to make a definition.

Definition 1.6. Let $n \geq 3$ be an odd integer. Let $C$ be an elliptic normal curve of degree $n$. A Klein matrix $\Phi$ for $C$ is an $n \times n$ alternating matrix of linear forms on $\mathbb{P}^{n-1}$ such that

(i) $\Phi$ has rank 2 at all points on $C$, and

(ii) the $n$ submaximal Pfaffians of $\Phi$ are linearly independent. 
We show in 6 that every Klein matrix is of the form $\Phi(\mathcal{E})$ for $\mathcal{E}$ an indecomposable rank 2 vector bundle on $C$ with $\operatorname{det} \mathcal{E} \simeq \mathcal{O}(1)$. Then Theorem 1.5(i) is a consequence of Atiyah's uniqueness result and the following existence theorem.

Theorem 1.7. Let $n \geq 3$ be an odd integer. Then every elliptic normal curve of degree $n$ has a Klein matrix.

In $\S \S 3$, 4 we give two independent proofs of Theorem 1.7 The first uses representations of the Heisenberg group and so is only valid if $\operatorname{char}(k) \nmid n$. The second uses the Buchsbaum-Eisenbud structure theorem for Gorenstein ideals of codimension 3, and hence applies over an arbitrary field. The latter may also be used to weaken Definition 1.6(i) to the statement that $\Phi$ has rank at most 2 on $C$.

Theorem 1.5 may be restated in terms of Klein matrices to give

Corollary 1.8. Let $n \geq 3$ be an odd integer. Let $C$ be an elliptic normal curve of degree $n$ with Klein matrix $\Phi$. If $n \geq 2 r+3$, then $\Phi$ is a Pfaffian presentation of $\operatorname{Sec}^{r} C$.

In future work we will study Klein matrices over a non-algebraically closed field. From the point of view of arithmetic, Pfaffian presentations are more interesting than determinantal presentations. Indeed it is possible for an elliptic normal curve $C$ of degree $n$ to have index $n$. This means that there are no $k$-rational divisors $D$ on $C$ with $0<\operatorname{deg} D<n$. In such circumstances it can be shown that $C$ has no determinantal presentations.

We recall some basic facts about Pfaffians. Let $A$ be an $n \times n$ alternating matrix over a ring $R$. This means that $A$ is skew-symmetric with zeros on the diagonal. If $n=2 m$ is even, then the Pfaffian $\operatorname{pf}(A)$ is a polynomial of degree $m$ in the entries of $A$ satisfying $\operatorname{pf}(A)^{2}=\operatorname{det}(A)$. In general the $2 m \times 2 m$ Pfaffians are the Pfaffians of the $2 m \times 2 m$ submatrices obtained by deleting the same rows and columns. They generate an ideal in $R$ that is unchanged if we replace $A$ by $P^{T} A P$ for $P \in \mathrm{GL}_{n}(R)$. In the case where $R$ is a field, the $(2 r+2) \times(2 r+2)$ Pfaffians of $A$ vanish if and only if $A$ has rank at most $2 r$. If $n$ is odd, then we call the $(n-1) \times(n-1)$ Pfaffians of $A$ the submaximal Pfaffians.

\section{Determinantal presentations}

The following lemma extends the work of Knight $\mathbf{K n}$, who considered divisors $D_{1}$ and $D_{2}$ that are multiples of a fixed point $P \in C$. The integers $\beta(r, n)$ were defined in Proposition 1.1 .

Lemma 2.1. Let $C$ be a smooth curve of genus one. Let $H$ be a divisor on $C$ of degree $n \geq 2$. Let $\mathcal{L}(H)$ have basis $x_{1}, \ldots, x_{n}$. Let $V_{r}$ be the vector space of $r$-ics in $k\left[x_{1}, \ldots, x_{n}\right]$ spanned by the $r \times r$ minors of all matrices $\Phi\left(D_{1}, D_{2}\right)$, as $D_{1}, D_{2}$ run over all divisors on $C$ with $D_{1}+D_{2}=H$ and $\operatorname{deg} D_{1}, \operatorname{deg} D_{2} \geq 1$. Then $\operatorname{dim} V_{r} \geq \beta(r, n)$.

Proof. We begin by treating the case $r=1$. Let $P_{1}$ and $P_{2}$ be distinct points on $C$ with $P_{1}+P_{2} \not H$. Then $\mathcal{L}(H)=\mathcal{L}\left(H-P_{1}\right)+\mathcal{L}\left(H-P_{2}\right)$ is spanned by the entries of $\Phi\left(P_{1}, H-P_{1}\right)$ and $\Phi\left(P_{2}, H-P_{2}\right)$. So $\operatorname{dim} V_{1}=n=\beta(1, n)$, as required.

The proof is now by induction on $r$ and $n$. The case $n<2 r$ is trivial since $\beta(r, n)=0$. We may therefore suppose that $r \geq 2$ and $n \geq 2 r$, and that the result is known for all smaller values of $r$ and $n$. 
Let $P \in C$ be any point. We may arrange that $x_{1}, \ldots, x_{n-i}$ is a basis for $\mathcal{L}(H-i P)$ for $i=0,1,2$. Let $g\left(x_{1}, \ldots, x_{n-2}\right)$ be an $(r-1) \times(r-1)$ minor of $\Phi\left(D_{1}, D_{2}\right)$, where $D_{1}+D_{2}=H-2 P$ and $\operatorname{deg} D_{1}, \operatorname{deg} D_{2} \geq 1$. It is determined by $(r-1)$-dimensional subspaces $W_{1} \subset \mathcal{L}\left(D_{1}\right)$ and $W_{2} \subset \mathcal{L}\left(D_{2}\right)$. Since $\operatorname{deg} D_{1}$, $\operatorname{deg} D_{2} \geq 1$ we may pick $w_{1} \in \mathcal{L}\left(D_{1}+P\right) \backslash \mathcal{L}\left(D_{1}\right)$ and $w_{2} \in \mathcal{L}\left(D_{2}+P\right) \backslash \mathcal{L}\left(D_{2}\right)$. Then $w_{1} w_{2} \in \mathcal{L}(H) \backslash \mathcal{L}(H-P)$. Rescaling $w_{1}$ if necessary we may assume that

$$
w_{1} w_{2}=x_{n}+\ell\left(x_{1}, \ldots, x_{n-1}\right),
$$

where $\ell$ is a linear form. Let $f$ be the $r \times r$ minor of $\Phi\left(D_{1}+P, D_{2}+P\right)$ determined by $W_{1} \oplus\left\langle w_{1}\right\rangle$ and $W_{2} \oplus\left\langle w_{2}\right\rangle$. Then

$$
f\left(x_{1}, \ldots, x_{n}\right)=x_{n} g\left(x_{1}, \ldots, x_{n-2}\right)+h\left(x_{1}, \ldots, x_{n-1}\right)
$$

for some $h \in k\left[x_{1}, \ldots, x_{n-1}\right]$. This construction of $f$ from $g$, combined with the induction hypothesis, shows that $V_{r} \cap k\left[x_{1}, \ldots, x_{n-1}\right]$ is a subspace of $V_{r}$ of codimension at least $\beta(r-1, n-2)$. But trivially, if $f\left(x_{1}, \ldots, x_{n-1}\right)$ is an $r \times r$ minor of $\Phi\left(D_{1}, D_{2}\right)$ with $D_{1}+D_{2}=H-P$, then it is also an $r \times r$ minor of $\Phi\left(D_{1}, D_{2}+P\right)$. Thus

$$
\operatorname{dim} V_{r} \geq \beta(r, n-1)+\beta(r-1, n-2)=\beta(r, n) .
$$

Let $C \subset \mathbb{P}^{n-1}$ be an elliptic normal curve with hyperplane section $H$. We noted in the Introduction that the $(r+1) \times(r+1)$ minors of $\Phi\left(D_{1}, D_{2}\right)$ vanish on $\operatorname{Sec}^{r} C$. So combining the last lemma with Proposition 1.1 gives

Lemma 2.2. If $n \geq 2 r+2$, then $I\left(\operatorname{Sec}^{r} C\right)$ is generated by the $(r+1) \times(r+1)$ minors of the matrices $\Phi\left(D_{1}, D_{2}\right)$, as $D_{1}, D_{2}$ run over all divisors on $C$ with $D_{1}+D_{2}=H$ and $\operatorname{deg} D_{1}, \operatorname{deg} D_{2} \geq 1$.

The aim of this section is to prove Theorem 1.3. This theorem is a variant of Lemma 2.2 giving necessary and sufficient conditions for $I\left(\operatorname{Sec}^{r} C\right)$ to be generated by the $(r+1) \times(r+1)$ minors of a single matrix $\Phi\left(D_{1}, D_{2}\right)$. First however we record a consequence of the results obtained so far that will be used in $\$ 4$. For any $P \in C$, we write $C_{P}$ and $C_{2 P}$ for the elliptic normal curves of degrees $n-1$ and $n-2$ obtained by projecting away from $P$ and $T_{P} C$.

Corollary 2.3. Let $n \geq 5$. If we choose co-ordinates on $\mathbb{P}^{n-1}$ so that $P \in C$ is the point $\left(x_{1}: \ldots: x_{n}\right)=(0: 0: \ldots: 0: 1)$, then

(i) $I\left(\operatorname{Sec}^{r} C\right) \cap k\left[x_{1}, \ldots, x_{n-1}\right]=I\left(\operatorname{Sec}^{r} C_{P}\right)$ and

(ii) for every $r$-ic $g \in I\left(\mathrm{Sec}^{r-1} C_{2 P}\right)$ there exists an $(r+1)-i c h \in k\left[x_{1}, \ldots, x_{n-1}\right]$ with $x_{n} g+h \in I\left(\operatorname{Sec}^{r} C\right)$.

Proof. (i) Let $\pi: \mathbb{P}^{n-1}-\rightarrow \mathbb{P}^{n-2} ;\left(x_{1}: \ldots: x_{n}\right) \mapsto\left(x_{1}: \ldots: x_{n-1}\right)$ be the projection map. Since $\operatorname{Sec}^{r} C_{P}$ is the Zariski closure of $\pi\left(\operatorname{Sec}^{r} C\right)$, the result is clear.

(ii) Let $H, H-P$ and $H-2 P$ be the hyperplane sections of $C, C_{P}$ and $C_{2 P}$. By Lemma 2.2 we may assume that $g$ is an $r \times r$ minor of $\Phi\left(D_{1}, D_{2}\right)$ for some $D_{1}$, $D_{2}$ with $D_{1}+D_{2}=H-2 P$ and $\operatorname{deg} D_{1}, \operatorname{deg} D_{2} \geq 1$. The proof of Lemma 2.1 constructs $f \in I\left(\operatorname{Sec}^{r} C\right)$, an $(r+1) \times(r+1)$ minor of $\Phi\left(D_{1}+P, D_{2}+P\right)$, with $f=x_{n} g+h$ for some $h \in k\left[x_{1}, \ldots, x_{n-1}\right]$.

Lemma 2.4. If $n \geq 2 r+3$, then $I\left(\operatorname{Sec}^{r} C\right)$ is generated by the ideals $I\left(\operatorname{Sec}^{r} C_{P}\right)$ as $P$ runs over any $n$ distinct points on $C$. 
Proof. Let $X$ be a subset of $C$ with $|X| \geq n$. Let $I$ be the ideal in $k\left[x_{1}, \ldots, x_{n}\right]$ generated by the $I\left(\operatorname{Sec}^{r} C_{P}\right)$ for $P \in X$. By Lemma 2.2 it suffices to show that if $D_{1}+D_{2}=H$, then all $(r+1) \times(r+1)$ minors of $\Phi\left(D_{1}, D_{2}\right)$ belong to $I$. Swapping $D_{1}$ and $D_{2}$ if necessary we may assume that $\operatorname{deg} D_{1} \geq r+2$. Let $d=\operatorname{deg} D_{1}$. We pick distinct points $P_{1}, \ldots, P_{d} \in X$ with $D_{1} \nsim P_{1}+\ldots+P_{d}$. Then

$$
\bigcap_{i=1}^{d} \mathcal{L}\left(D_{1}-P_{i}\right)=\mathcal{L}\left(D_{1}-\left(P_{1}+\ldots+P_{d}\right)\right)=0 .
$$

So there exists a basis $v_{1}, \ldots, v_{d}$ for $\mathcal{L}\left(D_{1}\right)$ such that $\mathcal{L}\left(D_{1}-P_{i}\right)$ has basis $v_{1}, \ldots, \widehat{v}_{i}$, $\ldots, v_{d}$. Then each $(r+1) \times(r+1)$ minor of $\Phi\left(D_{1}, D_{2}\right)$ is an $(r+1) \times(r+1)$ minor of $\Phi\left(D_{1}-P_{i}, D_{2}\right)$ for some $1 \leq i \leq d$. We are done, since the latter belong to $I\left(\operatorname{Sec}^{r} C_{P_{i}}\right)$.

Remark 2.5. The proof of Lemma 2.4 shows that it suffices for $P$ to run over any $n-r$ distinct points on $C$. This improvement is irrelevant for our applications.

For $D$ an effective divisor on $C$ we write $\bar{D}$ for the linear subspace of $\mathbb{P}^{n-1}$ cut out by $\mathcal{L}(H-D) \subset \mathcal{L}(H)$. If $D$ is a sum of distinct points, then $\bar{D}$ is simply the linear span of these points.

Lemma 2.6. Let $D, D_{1}, D_{2}$ be effective divisors on $C$. Then

(i) $\operatorname{dim} \bar{D}= \begin{cases}\operatorname{deg} D-1 & \text { if } \operatorname{deg} D<n, \\ n-2 & \text { if } D \sim H, \\ n-1 & \text { otherwise. }\end{cases}$

(ii) The linear span of $\overline{D_{1}}$ and $\overline{D_{2}}$ is $\overline{\mathrm{lcm}\left(D_{1}, D_{2}\right)}$.

(iii) $\overline{D_{1}} \cap \overline{D_{2}}=\overline{\operatorname{gcd}\left(D_{1}, D_{2}\right)}$ if $\operatorname{lcm}\left(D_{1}, D_{2}\right)$ has degree at most $n$ and is not linearly equivalent to $H$.

Proof. (i) This is immediate from Riemann-Roch.

(ii) We have $\mathcal{L}\left(H-D_{1}\right) \cap \mathcal{L}\left(H-D_{2}\right)=\mathcal{L}\left(H-\operatorname{lcm}\left(D_{1}, D_{2}\right)\right)$.

(iii) The inclusion " $\supset$ " is clear. Equality follows by counting dimensions using (i) and (ii).

Lemma 2.7. Let $D_{1}, D_{2}$ be divisors on $C$ with $D_{1}+D_{2}=H$ and $\operatorname{deg} D_{1} \leq \operatorname{deg} D_{2}$. Then

$$
\left\{\operatorname{rank} \Phi\left(D_{1}, D_{2}\right)<\operatorname{deg} D_{1}\right\}=\bigcup_{D \in\left|D_{1}\right|} \bar{D} .
$$

Proof. A point $P \in \mathbb{P}^{n-1}$ corresponds to a codimension 1 subspace $V_{P} \subset \mathcal{L}(H)$. If $\Phi\left(D_{1}, D_{2}\right)$ evaluated at $P$ has rank less than $\operatorname{deg} D_{1}$, then there exists non-zero $f \in \mathcal{L}\left(D_{1}\right)$ such that $f g \in V_{P}$ for all $g \in \mathcal{L}\left(D_{2}\right)$. Say $(f)=D-D_{1}$. Then $\mathcal{L}(H-D) \subset V_{P}$, so $P \in \bar{D}$. The converse is obtained by reversing these steps.

We make a temporary definition.

Definition 2.8. A divisor pair $\left(D_{1}, D_{2}\right)$ consists of divisors $D_{1}, D_{2}$ on $C$ with $D_{1}+D_{2}=H$ and $\operatorname{deg} D_{1}, \operatorname{deg} D_{2} \geq r+1$. We say that divisor pairs $\left(D_{1}, D_{2}\right)$ and $\left(D_{1}^{\prime}, D_{2}^{\prime}\right)$ are equivalent if $D_{1} \sim D_{1}^{\prime}$ or $D_{1} \sim D_{2}^{\prime}$.

The next lemma is a reworking of [R0, 9.22.1]. If $n=2 r+2$, then we already know by Proposition 1.1 that the space of $(r+1)$-ics vanishing on $\operatorname{Sec}^{r} C$ has dimension $\beta(r+1, n)=2$. 
Lemma 2.9 (Room). Let $n=2 r+2$ with $r \geq 1$. If $\left(D_{1}, D_{2}\right)$ and $\left(D_{1}^{\prime}, D_{2}^{\prime}\right)$ are inequivalent divisor pairs, then

$$
\operatorname{Sec}^{r} C=\left\{\operatorname{det} \Phi\left(D_{1}, D_{2}\right)=\operatorname{det} \Phi\left(D_{1}^{\prime}, D_{2}^{\prime}\right)=0\right\} \subset \mathbb{P}^{n-1} .
$$

Proof. As noted in the Introduction each of these $(r+1)$-ics contains $\operatorname{Sec}^{r} C$. Conversely, if $P$ belongs to the right hand side, we know by Lemma 2.7 that $P \in \bar{D} \cap \overline{D^{\prime}}$ for some $D \in\left|D_{1}\right|$ and $D^{\prime} \in\left|D_{1}^{\prime}\right|$. Since $D+D^{\prime} \nsim H$ it follows by Lemma 2.6(iii) that $P \in \overline{\operatorname{gcd}\left(D, D^{\prime}\right)}$. Since $D \neq D^{\prime}$, this shows that $P \in \operatorname{Sec}^{r} C$.

We strengthen Lemma 2.2 .

Lemma 2.10. If $n \geq 2 r+2$, then $I\left(\operatorname{Sec}^{r} C\right)$ is generated by the $(r+1) \times(r+1)$ minors of $\Phi\left(D_{1}, D_{2}\right)$ and $\Phi\left(D_{1}^{\prime}, D_{2}^{\prime}\right)$, where $\left(D_{1}, D_{2}\right)$ and $\left(D_{1}^{\prime}, D_{2}^{\prime}\right)$ are any two inequivalent divisor pairs.

Proof. The proof is by induction on $n$, the case $n=2 r+2$ having been treated in Lemma 2.9. We may therefore suppose that $n \geq 2 r+3$ and $\operatorname{deg} D_{1}, \operatorname{deg} D_{1}^{\prime} \geq r+2$. Let $P$ run over any $n$ distinct points on $C$ with $D_{1}-D_{2}^{\prime} \nsim P$. Then $\left(D_{1}-P, D_{2}\right)$ and $\left(D_{1}^{\prime}-P, D_{2}^{\prime}\right)$ are inequivalent divisor pairs on $C_{P} \subset \mathbb{P}^{n-2}$. By induction hypothesis the $(r+1) \times(r+1)$ minors of $\Phi\left(D_{1}-P, D_{2}\right)$ and $\Phi\left(D_{1}^{\prime}-P, D_{2}^{\prime}\right)$ generate $I\left(\operatorname{Sec}^{r} C_{P}\right)$. Since these are submatrices of $\Phi\left(D_{1}, D_{2}\right)$ and $\Phi\left(D_{1}^{\prime}, D_{2}^{\prime}\right)$, we are done by Lemma 2.4 .

Proof of Theorem 1.3. We may assume $\operatorname{deg} D_{1}, \operatorname{deg} D_{2} \geq r+1$ since there would otherwise be no $(r+1) \times(r+1)$ minors to consider. The proof is divided into 4 cases.

(i) Suppose that $\operatorname{deg} D_{1}, \operatorname{deg} D_{2} \geq r+2$ and $D_{1} \nsim D_{2}$. Let $P$ run over any $n$ distinct points on $C$. Then $\left(D_{1}-P, D_{2}\right)$ and $\left(D_{1}, D_{2}-P\right)$ are inequivalent divisor pairs on $C_{P} \subset \mathbb{P}^{n-2}$. We know by Lemma 2.10 that $I\left(\operatorname{Sec}^{r} C_{P}\right)$ is generated by the $(r+1) \times(r+1)$ minors of $\Phi\left(D_{1}-P, D_{2}\right)$ and $\Phi\left(D_{1}, D_{2}-P\right)$. Since these are submatrices of $\Phi\left(D_{1}, D_{2}\right)$, we are done by Lemma 2.4

(ii) Suppose that $\operatorname{deg} D_{1}, \operatorname{deg} D_{2} \geq r+3$ and $D_{1} \sim D_{2}$. Let $P$ run over any $n$ distinct points on $C$. We know by case (i) that $I\left(\operatorname{Sec}^{r} C_{P}\right)$ is generated by the $(r+1) \times(r+1)$ minors of $\Phi\left(D_{1}, D_{2}-P\right)$. Since this is a submatrix of $\Phi\left(D_{1}, D_{2}\right)$, we are done by Lemma 2.4 .

(iii) Suppose that $\operatorname{deg} D_{1}=r+1$ and $\operatorname{deg} D_{2} \geq r+1$. Then $\Phi\left(D_{1}, D_{2}\right)$ has at most $\left(\begin{array}{c}r+t+1 \\ t\end{array}\right)$ linearly independent minors where $t=\operatorname{deg} D_{2}-\operatorname{deg} D_{1}=n-2 r-2$. By Proposition 1.1 the vector space of $(r+1)$-ics generating $I\left(\operatorname{Sec}^{r} C\right)$ has dimension

$$
\beta(r+1, n)=\left(\begin{array}{c}
r+t+1 \\
t
\end{array}\right)+\left(\begin{array}{c}
r+t \\
t
\end{array}\right)>\left(\begin{array}{c}
r+t+1 \\
t
\end{array}\right) .
$$

Hence $\Phi\left(D_{1}, D_{2}\right)$ is not a determinantal presentation of $\operatorname{Sec}^{r} C$.

(iv) Suppose that $\operatorname{deg} D_{1}=\operatorname{deg} D_{2}=r+2$ and $D_{1} \sim D_{2}$. Choosing suitable bases for $\mathcal{L}\left(D_{1}\right)$ and $\mathcal{L}\left(D_{2}\right)$ we may arrange that $\Phi\left(D_{1}, D_{2}\right)$ is symmetric. Then $\Phi\left(D_{1}, D_{2}\right)$ has at most $(r+2)(r+3) / 2$ linearly independent minors. By Proposition 1.1 the vector space of $(r+1)$-ics generating $I\left(\operatorname{Sec}^{r} C\right)$ has dimension

$$
\beta(r+1, n)=(r+2)^{2}>(r+2)(r+3) / 2 .
$$

Hence $\Phi\left(D_{1}, D_{2}\right)$ is not a determinantal presentation of $\operatorname{Sec}^{r} C$. 


\section{The Heisenberg group}

Let $C$ be an elliptic normal curve of degree $n$. We prove Theorem 1.7 under the assumption char $(k) \nmid n$. We begin by describing the action of $E[n]$ on $C$, where $E$ is the Jacobian of $C$.

Lemma 3.1. Let $C \subset \mathbb{P}^{n-1}$ be an elliptic normal curve. Then $C$ meets each hyperplane in at most $n$ points. In the case of equality the points of intersection span the hyperplane.

Proof. Since $C$ is contained in no hyperplane, the first statement is Bézout's theorem. If the $n$ points of intersection fail to span the hyperplane, then we obtain a contradiction by considering another hyperplane through the same $n$ points and a further point of $C$.

Remark 3.2. An equally short proof of Lemma 3.1 uses Riemann-Roch instead of Bézout's theorem; cf. Lemma 2.6](i).

Lemma 3.3. Let $C \subset \mathbb{P}^{n-1}$ be an elliptic normal curve. Let $\tau_{P}$ be the translation map by $P \in E$, where $E$ is the Jacobian of $C$.

(i) The automorphism $\tau_{P}$ lifts to $\mathrm{PGL}_{n}$ if and only if $P \in E[n]$.

(ii) If $P$ has exact order $n$, then $\tau_{P}$ has exactly $n$ fixed hyperplanes.

Proof. (i) Let $H$ be the divisor of a hyperplane section. Then

$$
\tau_{P} \text { lifts to } \mathrm{PGL}_{n} \Longleftrightarrow \tau_{P}^{*} H \sim H \Longleftrightarrow P \in E[n] .
$$

(ii) We lift $\tau_{P} \in \mathrm{PGL}_{n}$ to $M_{P} \in \mathrm{GL}_{n}$ with $M_{P}^{n}=I_{n}$. Since char $(k) \nmid n$ we may assume that $M_{P}$ is diagonal, with each eigenvalue an $n$th root of unity. We consider a hyperplane fixed by $\tau_{P}$. The intersection of this hyperplane with $C$ is non-empty by Bézout's theorem and contains $n$ distinct points by the action of $\tau_{P}$. So by Lemma 3.1 the points of intersection span the hyperplane. Repeating for each fixed hyperplane, it follows that $M_{P}$ has no repeated eigenvalues. Equivalently $\tau_{P}$ has exactly $n$ fixed hyperplanes.

We fix $\zeta_{n} \in k$ a primitive $n$th root of unity. The Heisenberg group $H_{n}$ is the subgroup of $\mathrm{GL}_{n}$ generated by the matrices

$$
\theta(\sigma)=\left(\begin{array}{ccccc}
1 & 0 & 0 & \cdots & 0 \\
0 & \zeta_{n} & 0 & \cdots & 0 \\
0 & 0 & \zeta_{n}^{2} & \cdots & 0 \\
\vdots & \vdots & \vdots & & \vdots \\
0 & 0 & 0 & \cdots & \zeta_{n}^{n-1}
\end{array}\right), \quad \theta(\tau)=\left(\begin{array}{ccccc}
0 & 0 & \cdots & 0 & 1 \\
1 & 0 & \cdots & 0 & 0 \\
0 & 1 & \cdots & 0 & 0 \\
\vdots & \vdots & & \vdots & \vdots \\
0 & 0 & \cdots & 1 & 0
\end{array}\right) .
$$

As our notation indicates, we prefer to view $H_{n}$ as an abstract group with generators $\sigma$ and $\tau$. Then $\theta: H_{n} \rightarrow \mathrm{GL}_{n}$ is a faithful representation, called the Schrödinger representation. Notice that $H_{n}$ is a non-abelian group of order $n^{3}$ with centre generated by the commutator of $\sigma$ and $\tau$. Let $\bar{\theta}: H_{n} \rightarrow \mathrm{PGL}_{n}$ be the corresponding projective representation. We write $e_{n}: E[n] \times E[n] \rightarrow \mu_{n}$ for the Weil pairing.

Lemma 3.4. Let $C \subset \mathbb{P}^{n-1}$ be an elliptic normal curve with Jacobian $E$. Let $S, T \in E[n]$ with $e_{n}(S, T)=\zeta_{n}$. Then we may choose co-ordinates on $\mathbb{P}^{n-1}$ such that $\tau_{S}$ and $\tau_{T}$ are given by $\bar{\theta}(\sigma)$ and $\bar{\theta}(\tau)$. 
Proof. We know by Lemma 3.3 that $\tau_{S}$ and $\tau_{T}$ lift to $\mathrm{PGL}_{n}$ and that each has exactly $n$ fixed hyperplanes. Since $\tau_{S}$ and $\tau_{T}$ commute, the hyperplanes fixed by $\tau_{S}$ are permuted by $\tau_{T}$. Let $P$ belong to the intersection of $C$ and a hyperplane fixed by $\tau_{S}$. If the same hyperplane is fixed by a power of $\tau_{T}$, other than the identity, then the translates of $P$ under $E[n]$ give a contradiction to Lemma 3.1. It follows that $\tau_{T}$ cyclically permutes the $n$ hyperplanes fixed by $\tau_{S}$. Thus we may choose co-ordinates on $\mathbb{P}^{n-1}$ such that $\tau_{S}$ and $\tau_{T}$ are given by $\bar{\theta}(\sigma)$ and $\bar{\theta}(\tau)$, at least if we replace $\zeta_{n}$ by $\zeta_{n}^{r}$ for some $r$ coprime to $n$. In fact $\tau_{S}$ and $\tau_{T}$ commute in $\mathrm{PGL}_{n}$, but their commutator, when lifted to $\mathrm{GL}_{n}$, is independent of these liftings and gives one possible definition of the Weil pairing. It follows that $r=1$, as required.

Lemma 3.5. Let $C \subset \mathbb{P}^{n-1}$ be a Heisenberg invariant elliptic normal curve. Then $C$ is also invariant under

$$
\iota=\left(\begin{array}{ccccc}
1 & 0 & \cdots & 0 & 0 \\
0 & 0 & \cdots & 0 & 1 \\
0 & 0 & \cdots & 1 & 0 \\
\vdots & \vdots & & \vdots & \vdots \\
0 & 1 & \cdots & 0 & 0
\end{array}\right) .
$$

Proof. Let $P_{0} \in C$ with $n . P_{0} \sim H$, where $H$ is the hyperplane section. The automorphism $[-1]$ of the elliptic curve $\left(C, P_{0}\right)$ extends to $\mathbb{P}^{n-1}$ to give an involution $\iota \in \mathrm{PGL}_{n}$ satisfying $\iota \bar{\theta}(h) \iota=\bar{\theta}(h)^{-1}$ for all $h \in H_{n}$. Since $\bar{\theta}\left(H_{n}\right)$ is its own centraliser inside $\mathrm{PGL}_{n}$, it follows that $C$ is invariant under any involution satisfying these commutation relations. One such involution is given in the statement of the lemma.

Remark 3.6. In the case $k=\mathbb{C}$ we refer to $[\mathrm{H}$, Chapter I] for alternative proofs of Lemmas 3.4 and 3.5 using theta functions.

Following [F, Chapter 4] we derive equations for a Heisenberg invariant elliptic normal curve of odd degree. We write $\left(x_{0}: x_{1}: \ldots: x_{n-1}\right)$ for our co-ordinates on $\mathbb{P}^{n-1}$ and agree to read all subscripts $\bmod n$.

Proposition 3.7. Let $n \geq 5$ be an odd integer. Let $C$ be a Heisenberg invariant elliptic normal curve of degree $n$. Then $C$ contains a unique point of the form

$$
P_{0}=\left(0: a_{1}: a_{2}: \ldots:-a_{2}:-a_{1}\right)
$$

and has Klein matrix $\Phi=\left(a_{i-j} x_{i+j}\right)$.

Proof. Since $n$ is odd, there is a point $P_{0} \in C \cap\left\{x_{0}=0\right\}$ fixed by $\iota$. It takes the form

$$
\begin{aligned}
& P_{0}=\left(0: a_{1}: a_{2}: \ldots: a_{2}: a_{1}\right) \\
& \text { or } \quad P_{0}=\left(0: a_{1}: a_{2}: \ldots:-a_{2}:-a_{1}\right) \text {. } \quad(-)
\end{aligned}
$$

The $n^{2}$ translates of $P_{0}$ under $E[n]$ account for all intersections of $C$ with the coordinates hyperplanes $\left\{x_{i}=0\right\}$. It follows that the $a_{i}$ are non-zero for $i \neq 0$. We set $a_{0}=0$. The action of $\bar{\theta}(\sigma)$ now shows that $P_{0}$ is unique.

Let $W=H^{0}\left(C, \mathcal{O}_{C}(1)\right)$ be the space of linear forms on $\mathbb{P}^{n-1}$ and let $V \subset S^{2} W$ be the space of quadrics vanishing on $C$. By Proposition 1.1 we have $\operatorname{dim} V=$ $n(n-3) / 2$, whereas $\operatorname{dim} S^{2} W=n(n+1) / 2$. The action of $\bar{\theta}(\sigma)$ allows us to write $V=\oplus V_{i}$ and $S^{2} W=\oplus\left(S^{2} W\right)_{i}$ with

$$
V_{i} \subset\left(S^{2} W\right)_{i}=\left\langle x_{i}^{2}, x_{i-1} x_{i+1}, \ldots\right\rangle .
$$


Since $n$ is odd we deduce via the action of $\bar{\theta}(\tau)$ that $\operatorname{dim} V_{i}=(n-3) / 2$ and $\operatorname{dim}\left(S^{2} W\right)_{i}=(n+1) / 2$. Let $P_{0} \in C$ satisfy either $(+)$ or $(-)$. The translates of $P_{0}$ under $\bar{\theta}(\tau)$ impose some linear conditions on the coefficients of the quadrics in $V_{0}$. Since $V_{0} \subset\left(S^{2} W\right)_{0}$ has codimension 2 , it follows that $\operatorname{rank}\left(a_{i-j} a_{i+j}\right) \leq 2$. If $P_{0}$ is of the form $(+)$, then the top left $3 \times 3$ minor gives $2 a_{1}^{3} a_{2}^{2} a_{3}=0$. Since the $a_{i}$ are non-zero, it follows that $P_{0}$ must be of the form (-).

Now $\Phi=\left(a_{i-j} x_{i+j}\right)$ is an $n \times n$ alternating matrix of linear forms. Evaluated at $P_{0}$, it has rank 2 . So the $4 \times 4$ Pfaffians of $\Phi$ are quadrics vanishing at each of the $n^{2}$ translates of $P_{0}$ under $E[n]$. It follows by Bézout's theorem that these quadrics vanish on the whole of $C$. Hence $\Phi$ has rank 2 on $C$.

Deleting the first row and column of $\Phi$ gives a submaximal Pfaffian of the form $\pm\left(\prod_{i=1}^{(n-1) / 2} a_{i}\right) x_{0}^{(n-1) / 2}+\ldots$. Since the $a_{i}$ are non-zero, this Pfaffian is non-zero. We deduce via the action of the Heisenberg group that the $n$ submaximal Pfaffians of $\Phi$ are linearly independent. So $\Phi$ is a Klein matrix, as was to be shown.

This completes our proof of Theorem 1.7 under the assumption char $(k) \nmid n$. (Notice that the theorem is a tautology if $n=3$.) A little combinatorial checking shows that the Klein matrix $\Phi$ of Proposition 3.7 is a Pfaffian presentation of $C$. (Later this will follow as a special case of Corollary [1.8) The details are as follows.

Lemma 3.8. Let $n \geq 5$ be an odd integer. Let $\Gamma$ be the set of subsets of $\mathbb{Z} / n \mathbb{Z}$ of size 4 and sum 0 . Let $\Delta$ be the set of subsets of $(\mathbb{Z} / n \mathbb{Z}) /\{ \pm 1\}$ of size 3 . Then there is a surjective map

$$
f: \Gamma \rightarrow \Delta ; \quad\{i, j, k, l\} \mapsto\{i+j, i+k, i+l\} .
$$

Proof. We first check that $f$ is well-defined. Let $\gamma=\{i, j, k, l\} \in \Gamma$. If $i+j \equiv \pm(i+k)$ $(\bmod n)$, then $j \equiv k(\bmod n)$ or $i \equiv l(\bmod n)$. Since $i, j, k, l$ are distinct $\bmod n$, it follows that $f(\gamma)$ has size 3 . Moreover, since $i+j \equiv-(k+l)(\bmod n)$, the image of $\gamma$ is independent of the order in which we write its elements.

Now let $\gamma=\{i, j, k, l\}$ and $\gamma^{\prime}=\left\{i^{\prime}, j^{\prime}, k^{\prime}, l^{\prime}\right\}$. If $f(\gamma)=f\left(\gamma^{\prime}\right)$, then reordering the elements of $\gamma^{\prime}$ we may suppose $i+j \equiv i^{\prime}+j^{\prime}$ and $i+k \equiv i^{\prime}+k^{\prime}$. Then $i+l \equiv$ $\pm\left(i^{\prime}+l^{\prime}\right)$, giving either $(i, j, k, l)=\left(i^{\prime}, j^{\prime}, k^{\prime}, l^{\prime}\right)$ or $(i, j, k, l)=\left(-l^{\prime},-k^{\prime},-j^{\prime},-i^{\prime}\right)$. In an obvious notation $\gamma= \pm \gamma^{\prime}$. It is readily seen that $\gamma=-\gamma$ if and only if $0 \in f(\gamma)$. So for $\delta \in \operatorname{im} f, \# f^{-1}(\delta)=1$ or 2 , according to whether $0 \in \delta$ or $0 \notin \delta$. Surjectivity is established by counting:

$$
\frac{1}{n}\left(\begin{array}{l}
n \\
4
\end{array}\right)=\left(\begin{array}{c}
(n-1) / 2 \\
2
\end{array}\right)+2\left(\begin{array}{c}
(n-1) / 2 \\
3
\end{array}\right) .
$$

Let $C$ be a Heisenberg invariant elliptic normal curve of odd degree $n \geq 5$. Let $\Phi=\left(a_{i-j} x_{i+j}\right)$ and $V$ be as above. Then $C$ meets the co-ordinate hyperplanes $\left\{x_{i}=0\right\}$ in a total of $n^{2}$ distinct points. So every non-zero quadric in $V$ has at least 3 non-zero terms. Since $V_{0} \subset\left(S^{2} W\right)_{0}$ has codimension 2, it follows that, up to scalars, there is a unique quadric in $V_{0}$ involving any three of the monomials $x_{0}^{2}, x_{1} x_{n-1}, \ldots, x_{(n-1) / 2} x_{(n+1) / 2}$. These quadrics span $V_{0}$ and are indexed by $\Delta$. On the other hand the $4 \times 4$ Pfaffians of $\Phi$ belonging to $\left(S^{2} W\right)_{0}$ are indexed by $\Gamma$. So by Lemma 3.8 the $4 \times 4$ Pfaffians of $\Phi$ span $V$, and so generate $I(C)$.

Remark 3.9. The equations we derive for an elliptic normal curve, namely the $4 \times 4$ Pfaffians of $\Phi=\left(a_{i-j} x_{i+j}\right)$, appear in the work of Klein [Kl, §11] as relations among theta functions. This is our reason for calling $\Phi$ a Klein matrix. These equations, 
and their relationship with the modular curve $X(n)$, have also been studied in AR, [F], GP, [V]. Sometimes it is useful to consider the matrices $\left(a_{i-j} x_{i+j}\right)$ where $\left(a_{0}: a_{1}: \ldots: a_{n-1}\right)$ is any point on $C$. These are called Moore matrices; see ADHPR, GP.

\section{Gorenstein IDEALS}

We give an alternative proof of Theorem 1.7 that applies over an arbitrary field $k$. The idea is to replace our elliptic normal curve by a higher secant variety of codimension 3 and then apply the Buchsbaum-Eisenbud structure theorem.

Let $R=k\left[x_{1}, \ldots, x_{n}\right]$ be the homogeneous co-ordinate ring of $\mathbb{P}^{n-1}$, given its usual grading by degree. For $M=\bigoplus M_{d}$ a graded $R$-module we write $M(c)$ for the graded $R$-module with $M(c)_{d}=M_{c+d}$.

Theorem 4.1. Let $C \subset \mathbb{P}^{n-1}$ be an elliptic normal curve. If $m=n-2 r \geq 2$, then $I\left(\operatorname{Sec}^{r} C\right)$ has a minimal graded free resolution of the form

$$
\begin{array}{r}
0 \rightarrow R(-n) \rightarrow R(-n+r+1)^{b_{m-1}} \rightarrow R(-n+r+2)^{b_{m-2}} \\
\rightarrow \ldots \rightarrow R(-r-2)^{b_{2}} \rightarrow R(-r-1)^{b_{1}} \rightarrow R \rightarrow 0 .
\end{array}
$$

In particular $\operatorname{Sec}^{r} C$ is projectively Gorenstein of codimension $m$.

Proof. This was proved independently by Graf v. Bothmer and Hulek vBH, §8], and the author. See also GP for a discussion of the cases $r=1,2$.

The final statement follows from the form of the minimal free resolution and the fact, already noted in the Introduction, that $\operatorname{Sec}^{r} C \subset \mathbb{P}^{n-1}$ has codimension $m$. For this one uses the graded analogue of [E, Corollary 21.16]. One consequence (which also follows directly from the proofs cited above) is that $b_{m-i}=b_{i}$ for all $i$.

By $[\mathrm{BH}$, Theorem 4.1.15(a)] the Betti numbers are

$$
b_{i}(r, m)=\frac{m+2 r}{m+r-i} \prod_{j=r+1, j \neq r+i}^{m+r-1} \frac{j}{|r+i-j|} .
$$

Since $b_{1}(r, m)=\beta(r+1, m+2 r)$ we recognise Proposition 1.1 as a special case of Theorem 4.1. We are mainly interested in Theorem 4.1 in the cases $m=2,3$.

Proposition 4.2. Let $C \subset \mathbb{P}^{n-1}$ be an elliptic normal curve.

(i) If $n=2 r+2$, then $I\left(\operatorname{Sec}^{r} C\right)$ has a minimal graded free resolution of the form

$$
0 \longrightarrow R(-n) \longrightarrow R(-r-1)^{2} \longrightarrow R \longrightarrow 0 \text {. }
$$

(ii) If $n=2 r+3$, then $I\left(\operatorname{Sec}^{r} C\right)$ has a minimal graded free resolution of the form

$$
0 \longrightarrow R(-n) \stackrel{p^{T}}{\longrightarrow} R(-r-2)^{n} \stackrel{\Phi}{\longrightarrow} R(-r-1)^{n} \stackrel{p}{\longrightarrow} R \longrightarrow 0,
$$

where $\Phi$ is an $n \times n$ alternating matrix of linear forms and $p$ is the row vector of submaximal Pfaffians of $\Phi$.

Proof. This is a special case of Theorem 4.1. In case (ii) we use the BuchsbaumEisenbud structure theorem [BE1], BE2].

The next lemma shows how the theory of unprojection, first studied by Kustin and Miller [KM], can be used to deduce Proposition 4.2(ii) from Proposition 4.2(i). More importantly for us, it shows that the matrix $\Phi$ in Proposition 4.2 (ii) is a Klein matrix for $C$. 
Lemma 4.3. Let $n=2 r+3$ with $r \geq 1$. Let $I$ and $J$ be ideals in $R=k\left[x_{1}, \ldots, x_{n-1}\right]$ with minimal free resolutions

$$
F_{\bullet}: \quad 0 \longrightarrow R(-n+1) \stackrel{\left(\begin{array}{c}
-f_{2} \\
f_{1}
\end{array}\right)}{\longrightarrow} R(-r-1)^{2} \stackrel{\left(f_{1} f_{2}\right)}{\longrightarrow} R \longrightarrow 0
$$

and

$$
G_{\bullet}: \quad 0 \longrightarrow R(-n+2) \stackrel{g^{T}}{\longrightarrow} R(-r-1)^{n-2} \stackrel{\Psi}{\longrightarrow} R(-r)^{n-2} \stackrel{g}{\longrightarrow} R \longrightarrow 0,
$$

where $\Psi$ is an $(n-2) \times(n-2)$ alternating matrix of linear forms. Suppose that $\mathcal{I}$ is a prime ideal in $\mathcal{R}=R\left[x_{n}\right]$, with $\mathcal{I} \cap R=I$, generated by the entries of

$$
p=\left(f_{1}, f_{2}, x_{n} g_{1}+h_{1}, \ldots, x_{n} g_{n-2}+h_{n-2}\right)
$$

for some $h_{1}, \ldots, h_{n-2} \in R$ homogeneous of degree $r+1$. Then $\mathcal{I}$ has a minimal graded free resolution of the form

$$
\mathcal{F}_{\bullet}: \quad 0 \longrightarrow \mathcal{R}(-n) \stackrel{p^{T}}{\longrightarrow} \mathcal{R}(-r-2)^{n} \stackrel{\Psi^{\prime}}{\longrightarrow} \mathcal{R}(-r-1)^{n} \stackrel{p}{\longrightarrow} \mathcal{R} \longrightarrow 0,
$$

where

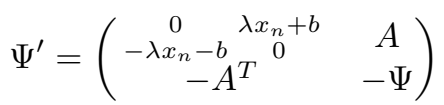

for some $0 \neq \lambda \in k, b \in R$ and $A \in \operatorname{Mat}_{2, n-2}(R)$.

Proof. Let $f, g$ and $h$ be the row vectors with entries $f_{i}, g_{i}$ and $h_{i}$. Since the entries of $h \Psi=\left(x_{n} g+h\right) \Psi$ belong to $\mathcal{I} \cap R=I$, there exists $A \in \operatorname{Mat}_{2, n-2}(R)$ with

$$
f A=h \Psi=\left(x_{n} g+h\right) \Psi .
$$

Post-multiplying by $g^{T}$ and using the exactness of $F_{\bullet}$ it follows that

$$
A g^{T}=\lambda\left(\begin{array}{c}
-f_{2} \\
f_{1}
\end{array}\right)
$$

for some $\lambda \in k$. We suppose for a contradiction that $\lambda=0$. Then (4.2) and the exactness of $G_{\bullet}$ give $A=D \Psi$ for some $D \in \operatorname{Mat}_{2, n-2}(k)$. Substituting in (4.1) and using the exactness of $G$ • once more gives $f D-h=\ell g$ for some linear form $\ell \in R$. Then $\left(x_{n}-\ell\right) g=\left(x_{n} g+h\right)-(\ell g+h)$ has entries in $\mathcal{I}$. Since $\mathcal{I}$ is a prime ideal generated in degree $r+1$, this forces $\ell=x_{n}$, contradicting the fact that $\ell \in R$.

By (4.2) we have

$$
A\left(x_{n} g+h\right)^{T}=\lambda x_{n}\left(\begin{array}{c}
-f_{2} \\
f_{1}
\end{array}\right)+A h^{T} .
$$

So the entries of $A h^{T}$ belong to $\mathcal{I} \cap R=I$, and there exists $B \in \operatorname{Mat}_{2,2}(R)$ with $A h^{T}=B f^{T}$. So by (4.1) and our hypothesis that $\Psi$ is alternating,

$$
f B f^{T}=f A h^{T}=h \Psi h^{T}=0 .
$$

Since $f_{1}, f_{2}$ are coprime, it follows that $B$ is alternating. So (4.3) becomes

$$
A\left(x_{n} g+h\right)^{T}=\left(\lambda x_{n}+b\right)\left(\begin{array}{c}
-f_{2} \\
f_{1}
\end{array}\right)
$$

for some linear form $b \in R$.

By (4.1) and (4.4) our resolution $\mathcal{F}_{\bullet}$ is a complex. It remains to prove it is exact at the terms $\mathcal{R}(-r-1)^{n}$ and $\mathcal{R}(-r-2)^{n}$. For the first of these we must show that if $(u, v) \in \mathcal{R}^{2} \times \mathcal{R}^{n-2}$ with

$$
u f^{T}+v\left(x_{n} g+h\right)^{T}=0
$$


then $(u, v)$ is an $\mathcal{R}$-linear combination of the rows of $\Psi^{\prime}$. We expand $u$ and $v$ in powers of $x_{n}$ as

$$
\begin{aligned}
& u=u_{p} x_{n}^{p}+\ldots+u_{1} x_{n}+u_{0}, \\
& v=v_{q} x_{n}^{q}+\ldots+v_{1} x_{n}+v_{0},
\end{aligned}
$$

where $u_{0}, \ldots, u_{p} \in R^{2}$ and $v_{0}, \ldots, v_{q} \in R^{n-2}$. The proof is by induction on $\max (2 p, 2 q+1)$, with the induction step divided into the following two cases. If $p>q$, then we subtract an $\mathcal{R}$-linear combination of the first two rows of $\Psi^{\prime}$ to decrease the value of $p$. If $p \leq q$, then we subtract an $\mathcal{R}$-linear combination of the last $n-2$ rows of $\Psi^{\prime}$ to decrease the value of $q$. (The latter is possible by comparing coefficients of $x_{n}^{q+1}$ in (4.5) and using the exactness of $G_{\bullet}$.) To start the induction we must treat the case $u \in R^{2}$ and $v=0$. But then exactness of $F_{\bullet}$ gives $u=\lambda t\left(-f_{2}, f_{1}\right)$ for some $t \in R$, and by (4.2) we have $u=\operatorname{tg} A^{T}$ and $v=\operatorname{tg} \Psi=0$, as required.

Finally we prove that $\mathcal{F}_{\bullet}$ is exact at the term $\mathcal{R}(-r-2)^{n}$. To do this we must show that if $(u, v) \in \mathcal{R}^{2} \times \mathcal{R}^{n-2}$ with $(u, v) \Psi^{\prime}=0$, then $(u, v)$ is a multiple of $p=\left(f, x_{n} g+h\right)$. We have $u A=v \Psi$, and so $u A g^{T}=0$. It follows by (4.2) and the exactness of $F_{\bullet}$ that $u$ is a multiple of $f$. This reduces us to the case $u=0$. Our hypothesis on $v$ is now that (i) $v A^{T}=0$ and (ii) $v \Psi=0$. By (ii) and the exactness of $G$. we see that $v$ is a multiple of $g$. Then (i) combined with (4.2) shows that $v=0$.

Proposition 4.4. Let $n \geq 3$ be an odd integer. Let $C$ be an elliptic normal curve of degree $n$. Let $\Phi$ be an $n \times n$ alternating matrix of linear forms. Then $\Phi$ is a Klein matrix for $C$ if and only if the submaximal Pfaffians of $\Phi$ generate $I\left(\operatorname{Sec}^{r} C\right)$ where $r=(n-3) / 2$.

Proof. Let $\Phi$ be a Klein matrix for $C$. Then $\Phi$ has rank 2 on $C$ and so has rank at most $2 r$ on $\operatorname{Sec}^{r} C$. So the submaximal Pfaffians of $\Phi$ belong to $I\left(\operatorname{Sec}^{r} C\right)$. Comparing dimensions in Definition 1.6 and Proposition 1.1 it follows that they generate $I\left(\operatorname{Sec}^{r} C\right)$.

Conversely suppose that the submaximal Pfaffians of $\Phi$ generate $I\left(\operatorname{Sec}^{r} C\right)$. By Proposition 1.1 they are linearly independent. It remains to show that $\Phi$ has rank 2 on $C$. The case $n=3$ being a tautology, we suppose $n \geq 5$. Let $P \in C$ be any point. We choose co-ordinates $\left(x_{1}: \ldots: x_{n}\right)$ on $\mathbb{P}^{n-1}$ so that $P=(0: 0: \ldots: 0: 1)$. Let $C_{P}$ and $C_{2 P}$ be the elliptic normal curves of degrees $n-1$ and $n-2$ obtained by projecting $C$ away from $P$ and $T_{P} C$. By Proposition 4.2, the ideals $I=I\left(\operatorname{Sec}^{r} C_{P}\right)$ and $J=I\left(\operatorname{Sec}^{r-1} C_{2 P}\right) R$ in $R=k\left[x_{1}, \ldots, x_{n-1}\right]$ have minimal free resolutions of the form specified in Lemma 4.3. We noted in the Introduction that $\operatorname{Sec}^{r} C$ is an irreducible variety. So $\mathcal{I}=I\left(\operatorname{Sec}^{r} C\right)$ is a prime ideal in $\mathcal{R}=R\left[x_{n}\right]$. The remaining hypotheses of Lemma 4.3 follow by Proposition 1.1 and Corollary 2.3.

The matrix $\Psi^{\prime}$ constructed in Lemma 4.3 has rank 2 when evaluated at $P=(0$ : $0: \ldots: 0: 1)$. By the Buchsbaum-Eisenbud structure theorem BE1, BE2, and the uniqueness of minimal free resolutions, the same is true for $\Phi$.

Combining Propositions 4.2 and 4.4 gives our second proof of Theorem 1.7 This not only establishes the existence of Klein matrices over an arbitrary field $k$ but also gives a practical algorithm for computing them.

Remark 4.5. We may weaken Definition 1.6(i) to the statement that $\Phi$ has rank at most 2 on $C$. This is equivalent to the original definition by Proposition 4.4. 


\section{Linear SeCtions of Grassmannians}

The Grassmannian $\operatorname{Gr}(2, m)=\operatorname{Gr}(2, V)$ is the set of 2-dimensional vector subspaces of an $m$-dimensional vector space $V$. It is an irreducible projective variety of dimension $2(m-2)$. The Plücker embedding is

$$
p: \operatorname{Gr}(2, V) \rightarrow \mathbb{P}\left(\bigwedge^{2} V\right) ; \quad\left\langle v_{1}, v_{2}\right\rangle \mapsto v_{1} \wedge v_{2}
$$

There is an exact sequence of vector bundles on $\operatorname{Gr}(2, V)$,

$$
0 \longrightarrow S \longrightarrow V^{*} \stackrel{\alpha}{\longrightarrow} Q \longrightarrow 0
$$

where $V^{*}$ is the trivial bundle of rank $m, S$ the universal sub-bundle of rank $m-2$, and $Q$ the universal quotient bundle of rank 2. The relationship between (5.1) and (5.2) is given by

Lemma 5.1. There is an isomorphism of line bundles $p^{*} \mathcal{O}(1) \simeq \operatorname{det} Q$ on $\operatorname{Gr}(2, V)$ and a commutative diagram

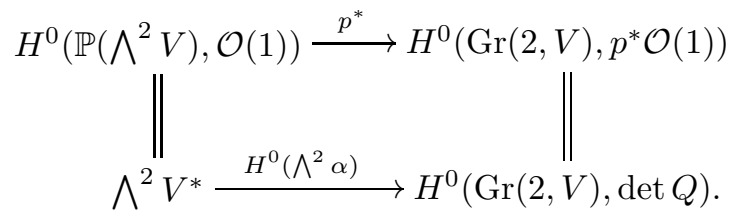

Now let $C$ be a smooth projective curve embedded by a complete linear system. As in the Introduction we write $\Phi(\mathcal{E})$ for the matrix of linear forms representing the determinant map on global sections for a rank 2 vector bundle $\mathcal{E}$ on $C$ with $\operatorname{det} \mathcal{E} \simeq \mathcal{O}(1)$

Lemma 5.2. Let $\Phi$ be an alternating matrix of linear forms. If $\Phi$ has rank 2 on $C$, then there exists a rank 2 vector bundle $\mathcal{E}$ on $C$ with $\operatorname{det} \mathcal{E} \simeq \mathcal{O}(1)$ and a matrix $B$ with entries in $k$ such that $\Phi=B^{T} \Phi(\mathcal{E}) B$.

Proof. Let $\phi: C \rightarrow \mathbb{P}\left(\bigwedge^{2} V\right)$ be the morphism determined by $\Phi$. Since $\Phi$ has rank 2 on $C$, this morphism factors via the Plücker embedding, say $\phi=p \pi$ for some $\pi: C \rightarrow \operatorname{Gr}(2, V)$. Let $\mathcal{E}=\pi^{*} Q$. Then $\mathcal{E}$ is a rank 2 vector bundle on $C$. Since the entries of $\Phi$ are linear forms, we have $\phi^{*} \mathcal{O}(1)=\mathcal{O}(1)$. So by Lemma 5.1,

$$
\operatorname{det} \mathcal{E} \simeq \pi^{*}(\operatorname{det} Q) \simeq \pi^{*} p^{*} \mathcal{O}(1) \simeq \mathcal{O}(1) .
$$

We obtain a commutative diagram

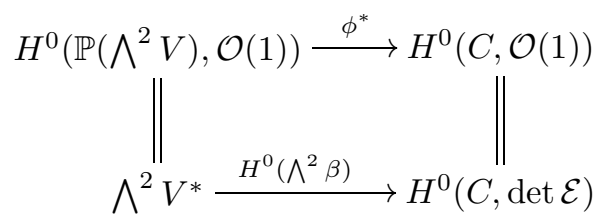

where $\beta=\pi^{*}(\alpha): V^{*} \rightarrow \mathcal{E}$. We are done since $\phi^{*}$ is represented by $\Phi$ and $H^{0}\left(\bigwedge^{2} \beta\right)$ is represented by $B^{T} \Phi(\mathcal{E}) B$, where $B$ is the matrix representing the linear map $H^{0}(\beta): V^{*} \rightarrow H^{0}(C, \mathcal{E})$.

We describe the vector bundles that can arise in the case where $C$ is an elliptic normal curve. 
Lemma 5.3. Let $C$ be an elliptic normal curve of degree $n$. Suppose that $C$ is the rank 2 locus of an alternating matrix of linear forms $\Phi$. Then the rank 2 vector bundle of Lemma 5.2 is either indecomposable or of the form $\mathcal{L}_{1} \oplus \mathcal{L}_{2}$ with $\operatorname{deg} \mathcal{L}_{1}, \operatorname{deg} \mathcal{L}_{2} \geq 3$.

Proof. Suppose that $\mathcal{E}$ is decomposable, say $\mathcal{E} \simeq \mathcal{O}\left(D_{1}\right) \oplus \mathcal{O}\left(D_{2}\right)$ with $D_{1}+D_{2}=H$. Then $C$ is the rank 1 locus of $\Phi\left(D_{1}, D_{2}\right)$. Since $C \neq \mathbb{P}^{n-1}$, we have $\operatorname{deg} D_{1}, \operatorname{deg} D_{2} \geq$ 2. If $\operatorname{deg} D_{1}=2$, then $C$ is a $\mathbb{P}^{n-1}$-section of the image of the Segre embedding $\mathbb{P}^{1} \times \mathbb{P}^{n-3} \hookrightarrow \mathbb{P}^{2 n-5}$. So $C$ has dimension at least $(n-1)+(n-2)-(2 n-5)=2$. This is a contradiction.

\section{Pfaffian presentations}

We recall a theorem of Atiyah.

Proposition 6.1. Let $C$ be a smooth curve of genus one. Let $(r, d)=1$ and let $\mathcal{L}$ be a line bundle on $C$ of degree $d$. Then there is a unique indecomposable rank $r$ vector bundle $\mathcal{E}$ on $C$ with determinant $\mathcal{L}$.

Proof. See [A, Corollary to Theorem 7].

The degree of a vector bundle is by definition the degree of its determinant. We will use the following explicit form of Riemann-Roch.

Lemma 6.2. Let $C$ be a smooth curve of genus one. Let $\mathcal{E}$ be an indecomposable vector bundle on $C$. If $\operatorname{det} \mathcal{E} \nsucceq \mathcal{O}$, then $\operatorname{dim} H^{0}(C, \mathcal{E})=\max (0, \operatorname{deg} \mathcal{E})$.

Proof. See [A, Lemmas $\left.6^{\prime}, 15\right]$.

Proposition 6.3. Let $n \geq 3$ be an odd integer. Let $C$ be an elliptic normal curve of degree $n$. Let $\Phi$ be an $n \times n$ alternating matrix of linear forms on $\mathbb{P}^{n-1}$. Then $\Phi$ is a Klein matrix for $C$ if and only if $\Phi=\Phi(\mathcal{E})$ for some indecomposable rank 2 vector bundle $\mathcal{E}$ on $C$ with $\operatorname{det} \mathcal{E} \simeq \mathcal{O}(1)$.

Proof. Let $\Phi$ be a Klein matrix for $C$. By Lemma 5.2 we have $\Phi=B^{T} \Phi(\mathcal{E}) B$, where $B$ is a matrix with entries in $k$. By Definition 1.6 the $(n-1) \times(n-1)$ Pfaffians of $\Phi$ span a vector space of dimension at least $n$. The same must therefore be true of $\Phi(\mathcal{E})$.

We suppose for a contradiction that $\mathcal{E}$ is decomposable, say

$$
\mathcal{E} \simeq \mathcal{O}\left(D_{1}\right) \oplus \mathcal{O}\left(D_{2}\right)
$$

with $D_{1}+D_{2}=H$. Then the $(n-1) \times(n-1)$ Pfaffians of $\Phi(\mathcal{E})$ are the $(n-$ $1) / 2 \times(n-1) / 2$ minors of $\Phi\left(D_{1}, D_{2}\right)$. If $n \geq 5$, then there are no such minors unless $\operatorname{deg} D_{1}=(n-1) / 2$ and $\operatorname{deg} D_{2}=(n+1) / 2$. But even in that case there are at most $(n+1) / 2$ linearly independent minors. This is the required contradiction.

In the case $n=3$ we must also rule out the possibility $\mathcal{E} \simeq \mathcal{O} \oplus \mathcal{O}(1)$. It suffices to note that there are no $4 \times 3$ matrices $B$ over $k$ for which

$$
B^{T}\left(\begin{array}{cccc}
0 & x_{1} & x_{2} & x_{3} \\
-x_{1} & 0 & 0 & 0 \\
-x_{2} & 0 & 0 & 0 \\
-x_{3} & 0 & 0 & 0
\end{array}\right) B
$$

has 3 linearly independent entries. 
We have established that $\mathcal{E}$ is indecomposable. Lemma 6.2 gives $\operatorname{dim} H^{0}(C, \mathcal{E})=$ $n$. So $\Phi(\mathcal{E})$ is an $n \times n$ alternating matrix of linear forms. The linear independence of the submaximal Pfaffians of $\Phi$ implies that $B$ is invertible. Since in the definition of $\Phi(\mathcal{E})$ we made an arbitrary choice of basis for $H^{0}(C, \mathcal{E})$, we are now free to suppose that $B$ is the identity matrix. Hence $\Phi=\Phi(\mathcal{E})$.

The converse follows by Atiyah's uniqueness result (Proposition 6.1) and the existence of Klein matrices (Theorem 1.7).

Proof of Theorem 1.5. Let $C$ be an elliptic normal curve of degree $n$ and let $\mathcal{E}$ be the unique indecomposable rank 2 vector bundle on $C$ with $\operatorname{det} \mathcal{E} \simeq \mathcal{O}(1)$.

(i) The matrix $\Phi(\mathcal{E})$ is a Klein matrix by Proposition 6.3. So by definition its submaximal Pfaffians are linearly independent.

(ii) It is clear that $\Phi(\mathcal{E})$ has rank at most 2 on $C$ and so has rank at most $2 r$ on $\operatorname{Sec}^{r} C$. So the $(2 r+2) \times(2 r+2)$ Pfaffians of $\Phi(\mathcal{E})$ belong to $I\left(\operatorname{Sec}^{r} C\right)$. We must show that they generate this ideal. If $n=2 r+3$, then we are done by (i) and Proposition 1.1.

The proof is now by induction on odd values of $n$. Accordingly we suppose $n \geq 2 r+5$ and that the result is known for $n-2$. Let $D$ be any effective divisor on $C$ of degree 2. Let $C_{D} \subset \mathbb{P}^{n-3}$ be the elliptic normal curve of degree $n-2$ obtained by projecting away from $D$. There is a natural inclusion $I\left(\operatorname{Sec}^{r} C_{D}\right) \subset I\left(\operatorname{Sec}^{r} C\right)$ where these ideals belong to different rings. By Lemma 2.4 it suffices to find generators for $I\left(\operatorname{Sec}^{r} C_{D}\right)$ among the $(2 r+2) \times(2 r+2)$ Pfaffians of $\Phi(\mathcal{E})$.

Let $P \in C$ with $D \sim 2 P$, and let $\mathcal{E}_{P}=\mathcal{E} \otimes \mathcal{O}(-P)$. Then

$$
\operatorname{det} \mathcal{E}_{P} \simeq(\operatorname{det} \mathcal{E}) \otimes \mathcal{O}(-2 P) \simeq \mathcal{O}(H-D),
$$

where $H$ and $H-D$ are the hyperplane sections for $C$ and $C_{D}$. By induction hypothesis $\Phi\left(\mathcal{E}_{P}\right)$ is a Pfaffian presentation of $\operatorname{Sec}^{r} C_{D}$. We are done since $\Phi\left(\mathcal{E}_{P}\right)$ is a submatrix of $\Phi(\mathcal{E})$.

Corollary 1.8 now follows from Proposition 6.3 and Theorem 1.5. In the case where $n=5$ we may characterise Klein matrices more simply as follows.

Corollary 6.4. Let $C \subset \mathbb{P}^{4}$ be an elliptic normal quintic. Let $\Phi$ be a $5 \times 5$ alternating matrix of linear forms on $\mathbb{P}^{4}$. Then $\Phi$ is a Klein matrix for $C$ if and only if $C$ is the rank 2 locus of $\Phi$.

Proof. Let $\Phi$ be a Klein matrix for $C$. Taking $r=1$ in Corollary 1.8 shows that $C$ is the rank 2 locus of $\Phi$.

Conversely, if $C$ is the rank 2 locus of $\Phi$, we must show that the $4 \times 4$ Pfaffians of $\Phi$ are linearly independent. By Lemmas 5.2 and 5.3 we have $\Phi=B^{T} \Phi(\mathcal{E}) B$, where $\mathcal{E}$ is an indecomposable rank 2 vector bundle on $C$ with $\operatorname{det} \mathcal{E} \simeq \mathcal{O}(1)$. Then Lemma 6.2 gives $\operatorname{dim} H^{0}(C, \mathcal{E})=5$. So $\Phi(\mathcal{E})$ is a $5 \times 5$ alternating matrix of linear forms. If $B$ were singular, then the $4 \times 4$ Pfaffians of $\Phi$ would belong to a 1dimensional vector space. But $C$ cannot be defined by a single quadric. So $B$ is invertible and $\Phi$ is a Klein matrix by Proposition 6.3 .

The analogue of Corollary 6.4 for $n \geq 7$ is false. To see this let $D_{1}, D_{2}$ be divisors on $C$ with $D_{1}+D_{2}=H$ and $\operatorname{deg} D_{1}$, deg $D_{2} \geq 3$. Put $\mathcal{E}=\mathcal{O}\left(D_{1}\right) \oplus \mathcal{O}\left(D_{2}\right)$. Then $C$ is the rank 2 locus of $\Phi(\mathcal{E})$ by Theorem 1.3 , but $\Phi(\mathcal{E})$ is not a Klein matrix for $C$. 


\section{REFERENCES}

[AR] A. Adler, S. Ramanan, Moduli of abelian varieties, Lecture Notes in Mathematics, 1644, Springer-Verlag, Berlin, 1996. MR1621185 (2000b:14057)

[A] M.F. Atiyah, Vector bundles over an elliptic curve, Proc. London Math. Soc. (3) 7 (1957) 414-452. MR0131423(24:A1274)

[ADHPR] A. Aure, W. Decker, K. Hulek, S. Popescu, K. Ranestad, Syzygies of abelian and bielliptic surfaces in $\mathbb{P}^{4}$, Internat. J. Math. 8 (1997), no. 7, 849-919. MR 1482969 (99a:14049)

[BH] W. Bruns, J. Herzog, Cohen-Macaulay rings, Cambridge Studies in Advanced Mathematics 39, Cambridge University Press, 1993. MR1251956 (95h:13020)

[BE1] D.A. Buchsbaum and D. Eisenbud, Gorenstein ideals of height 3, Seminar D. Eisenbud/B. Singh/W. Vogel, Vol. 2, pp. 30-48, Teubner-Texte zur Math., 48, Teubner, Leipzig, 1982. MR686456 (84i:13017)

[BE2] D.A. Buchsbaum and D. Eisenbud, Algebra structures for finite free resolutions, and some structure theorems for ideals of codimension 3, Amer. J. Math. 99 (1977) 447-485. MR0453723 (56:11983)

[E] D. Eisenbud, Commutative algebra with a view toward algebraic geometry, GTM 150, Springer-Verlag, New York, 1995. MR,1322960 (97a:13001)

[EKS] D. Eisenbud, J. Koh, M. Stillman, Determinantal equations for curves of high degree, Amer. J. Math. 110 (1988), no. 3, 513-539. MR944326 (89g:14023)

[F] T.A. Fisher, On 5 and 7 descents for elliptic curves, Ph.D. thesis, University of Cambridge, 2000.

[GP] M. Gross, S. Popescu, Equations of $(1, d)$-polarized abelian surfaces, Math. Ann. 310 (1998), no. 2, 333-377. MR1602020 (99d:14046)

[H] K. Hulek, Projective geometry of elliptic curves, Soc. Math. de France, Astérisque 137 (1986). MR845383 (88c:14046)

[Kl] F. Klein, Über die elliptischen Normalkurven der $n$-ten Ordnung (1885), in Gesammelte Mathematische Abhandlungen, 3: Elliptische Funktionen etc., R. Fricke et al. (eds.), Springer (1923).

[Kn] A.J. Knight, Primals passing multiply through elliptic normal curves, Proc. London Math. Soc. (3) 23 (1971), 445-458. MR0291173 (45:267)

[KM] A.R. Kustin, M. Miller, Constructing big Gorenstein ideals from small ones, J. Algebra 85 (1983), no. 2, 303-322. MR725084 (85f:13014)

[L] H. Lange, Higher secant varieties of curves and the theorem of Nagata on ruled surfaces, Manuscripta Math. 47 (1984), no. 1-3, 263-269. MR744323 (85f:14043)

[Ra] M.S. Ravi, Determinantal equations for secant varieties of curves, Comm. Algebra 22 (1994), no. 8, 3103-3106. MR.1272376 (95c:14029)

[Ro] T.G. Room, The geometry of determinantal loci, Cambridge University Press, 1938.

[vBH] H.-Chr. Graf v. Bothmer, K. Hulek, Geometric syzygies of elliptic normal curves and their secant varieties, Manuscripta Math. 113 (2004), no. 1, 35-68. MR2135560 (2006b:14009)

[V] J. Vélu, Courbes elliptiques munies d'un sous-groupe $\mathbb{Z} / n \mathbb{Z} \times \mu_{n}$, Bull. Soc. Math. France Mém. No. 57 (1978), 5-152. MR507751 (80a:14011)

Department of Pure Mathematics and Mathematical Statistics, Centre for Mathematical Sciences, University of Cambridge, Wilberforce Road, Cambridge CB3 0WB, United Kingdom

E-mail address: T.A.Fisher@dpmms.cam.ac.uk 